\title{
Phytoestrogen supplementation and body composition in postmenopausal women: A systematic review and meta-analysis of randomized controlled trials
}

\author{
Marija Glisic $^{\mathrm{a}, *}$, Natyra Kastrati ${ }^{\mathrm{a}, 1}$, Juna Musa ${ }^{\mathrm{a}, 1}$, Jelena Milic ${ }^{\mathrm{a}}$, Eralda Asllanaj ${ }^{\mathrm{a}}$, \\ Eliana Portilla Fernandez ${ }^{\mathrm{a}}$, Jana Nano ${ }^{\mathrm{a}}$, Carolina Ochoa Rosales ${ }^{\mathrm{a}, \mathrm{b}}$, Masoud Amiri ${ }^{\mathrm{a}, \mathrm{c}}$, \\ Bledar Kraja ${ }^{a}$, Arjola Bano ${ }^{a}$, Wichor M. Bramer ${ }^{d}$, Anton J.M. Roks ${ }^{\mathrm{e}}$, A.H. Jan Danser ${ }^{\mathrm{e}}$, \\ Oscar H. Franco ${ }^{\mathrm{a}, \mathrm{f}}$, Taulant Muka ${ }^{\mathrm{a}, \mathrm{g}}$ \\ a Department of Epidemiology, Erasmus MC, University Medical Center Rotterdam, The Netherlands \\ ${ }^{\mathrm{b}}$ Centro de Vida Saludable de la Universidad de Concepción, Concepción, Chile \\ ${ }^{\mathrm{c}}$ Department of Epidemiology and Biostatistics, Shahrekord University of Medical Sciences, Shahrekord, Iran \\ d Medical Library, Erasmus MC, University Medical Center, Rotterdam, The Netherlands \\ e Department of Internal Medicine, Division of Vascular Medicine and Pharmacology, Erasmus MC, University Medical Center Rotterdam, The Netherlands \\ ${ }_{\mathrm{f}}^{\mathrm{I}}$ Institute of Social and Preventive Medicine (ISPM), University of Bern, Bern, Switzerland \\ ${ }^{\mathrm{g}}$ Almedicus, Tirana, Albania
}

\section{A R T I C L E I N F O}

\section{Keywords:}

Phytoestrogen supplementation

Body composition

Menopausal women

Systematic review

Meta-analysis

Randomized controlled trials

\begin{abstract}
A B S T R A C T
Phytoestrogen-based medications are commonly used by menopausal women, and especially by obese postmenopausal women, to relieve menopausal symptoms. Substitution of animal with soy protein is often used in weight loss regimens, yet the effect of phytoestrogens, the main constituent of soy foods, on body composition is not completely understood. We conducted a systematic review and meta-analysis to investigate the associations between phytoestrogen supplementation and body weight and the main parameters of body composition in postmenopausal women. A literature search was done using 5 electronic databases from inception to April 2018. Randomized controlled trials (RCTs) with postmenopausal women comparing phytoestrogen supplementation followed by usual diet and placebo were included in the present meta-analysis. From 5932 references, we identified 23 RCTs that met our inclusion criteria, with a total of 1880 postmenopausal women. No association was observed between phytoestrogen supplementation and body weight, body mass index, waist and hip circumference, total fat mass or percentage of body fat. However, the use of phytoestrogens supplementation was associated with a slight decrease in waist-hip ratio; the pooled mean difference was $-0.01 \mathrm{~cm}(95 \% \mathrm{CI}:-0.01$ to -0.006). In subgroup analysis, we found a modest decrease in body weight with phytoestrogens supplementation compared with placebo in healthy postmenopausal women [pooled mean difference of changes $-0.28 \mathrm{~kg}$ (95\%CI: -0.52 to -0.04$)$ ] and in RCTs with a median number of participants of 66 or less [pooled mean difference of changes $-0.49 \mathrm{~kg}$ (95\%CI: -0.87 to -0.11$)$ ]. In contrast, phytoestrogen supplementation was associated with increased body weight in postmenopausal women with preexisting metabolic disorders (prediabetes, type 2 diabetes, prehypertension and hyperlipidemia) [pooled mean difference of changes: $0.78 \mathrm{~kg}$ (95\%CI: 0.53-1.03)]. In addition, there were some indications that some types of phytoestrogens, such as daidzein, but not soy products or isoflavone mix, could lead to modest adverse changes in body composition in menopausal women. Therefore, future studies should investigate the potential adverse effects of phytoestrogen supplementation on body composition among postmenopausal women.
\end{abstract}

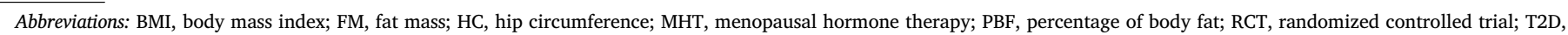
type 2 diabetes; WC, waist circumference; WHR, waist to hip ratio

* Corresponding author at: Department of Epidemiology, Erasmus University Medical Center, Dr. Molewaterplein 50, Office NA29-09, PO Box 2040, 3000 CA Rotterdam, The Netherlands.

E-mail address: m.glisic@erasmusmc.nl (M. Glisic).

${ }^{1}$ Equally contributed. 


\section{Introduction}

Menopause is considered as the end of woman's reproductive life, generally defined as "cessation" of menstrual periods for twelve consecutive months [1]. Menopausal transition is characterized by hormonal disturbances, irregular menstrual periods and presence of menopausal symptoms [1]. The most challenging menopausal symptoms include hot flashes and night sweats, with $50.3 \%-82.1 \%$ of postmenopausal reporting either mild, moderate or severe vasomotor symptoms [2,3]. In women, aging and menopause-induced estrogen deficiency could result in an increase in body weight and may lead to abdominal fat accumulation and decrease in lean mass during menopausal transition [4]. Overweight and obese menopausal women may also tend to have more prevalent [8] and more severe menopausal symptoms [5-8]. Thus, menopausal hormone therapy (MHT) remains the most effective treatment for menopausal vasomotor symptoms [9]. However, given the potentially undesirable health consequences of hormone therapy on cardiovascular health and breast cancer risk, the number of women choosing plant based-therapies as an alternative to treat menopausal symptoms is increasing [10].

The most commonly used herbal therapies may include "over the counter" phytoestrogen supplements, such as dietary soy isoflavones and soy extracts and herbal remedies such as red clover and black cohosh [10]. Phytoestrogens are a group of biologically active plant-derived compounds with estrogen-like properties [11]. Isoflavones (genisten and diadzein) and lignans are the most used phytoestrogens; while isoflavones can be abundantly found in soybeans, lignans are found in legumes, vegetables, fruits, flaxseed and whole grains [11]. Recent meta-analysis of clinical trials showed that specific phytoestrogen supplementation led to relief of menopausal symptoms [10]. Nevertheless, there is inconsistent evidence whether phytoestrogens could additionally help to reduce body weight and counteract the adverse changes that may occur in body composition in women after menopause. While few studies indicated that phytoestrogens may lead to modest improvements in body weight and the other parameters of body composition [12-14], there were few studies raising concerns that phytoestrogens could lead to adverse body composition changes, such as increase in weight [15-18] and body mass index (BMI) [16,18-20]. A meta-analysis of nine randomized trials (conducted in 2013) has suggested that isoflavone supplementation might reduce the body weight [21]. However, this study was limited only to non-Asian postmenopausal women and by investigating only the changes in body weight and not the other parameters of body composition. Furthermore, only trials that investigated solely isoflavone supplementation, and not the other types of phytoestrogens, were included in that review.

Therefore, this comprehensive systematic review and meta-analysis of intervention studies aimed to evaluate the association between phytoestrogen supplementation followed with regular/normocaloric diet and body composition in postmenopausal women.

\section{Methods}

\subsection{Data sources and search strategy}

The Cochrane Handbook for Systematic Reviews of Interventions and PRISMA Statement were used to guide the conduct and reporting of this review [22]. A literature search was done using 5 electronic databases (Medline via Ovid, EMBASE, Web of Science Core Collection, Cochrane CENTRAL via Wiley and Google Scholar) from inception to April 2018 (date last searched). Additionally, we searched the reference lists of the included studies and relevant reviews. Details on the search strategy are provided in Supplemental Table 1.

\subsection{Study selection and data extraction}

Studies were included if they met the following inclusion criteria: (i) were randomized placebo-controlled trials (ii) reported associations of phytoestrogen supplementation with total body weight, total fat mass (FM), percentage of body fat (PBF), BMI, waist circumference (WC), and waist to hip ratio (WHR), (iii) were performed among postmenopausal women and (iv) investigated phytoestrogen supplementation in intervention arm alone. RCTs investigated combination of exposures (e.g. hypocaloric diet or exercise with phytoestrogen supplementation) were not included in this study. Additionally, as we were interested to evaluate overall effect of phytoestrogens on body weight (and not its effect on weight loss), we decided to include RCTs irrespectively of the study aim; therefore, all eligible RCTs that reported baseline and end of study information on outcomes of interest were included in this review. Two reviewers independently evaluated the titles and abstracts according to the inclusion and exclusion criteria. For each potentially eligible study, two reviewers assessed the full-text. In cases of disagreement, a decision was made by consensus or, if necessary, a third reviewer was consulted. A predesigned data extraction form was used to collect relevant information.

\subsection{Assessing the risk of bias}

The risk of bias within each individual study was evaluated by two reviewers. To assess the quality of RCTs, "The Cochrane Collaboration's tool" for assessing risk of bias was used [23]. Studies were judged to be at lower high risk of bias based on criteria to evaluate random sequence generation, allocation concealment, blinding of participants/personnel and outcome assessment, incomplete outcome data and selective reporting [23]. RCTs were considered to be in low risk of bias, if allocation concealment, blinding of participants and outcome assessors were all coded "yes", if a compliance assessment was done, and the number of dropouts and reasons for dropout were reported, otherwise the RCTs were considered to be at high risk of bias. If the risk of bias couldn't be determined in any of the segments (e.g. information not provided) the risk of bias was classified as unknown (Supplemental Table 2).

\subsection{Data synthesis and analysis}

Summary outcomes measures were presented as mean differences (intervention minus control) of the treatment effects (differences in outcomes at the end of trial) between treatment groups in body weight, BMI, WC, HC, HC, FM and PBF. Estimates of weighted mean differences (WMDs) and 95\% CIs were obtained using random-effect model. In case of cross-over trials, the data from the first period only were used. In addition, for sensitivity analysis, the estimates were reported using fixed effect models as shown in the forest plots. Heterogeneity was quantified using the $I^{2}$ statistic, classified as low $\left(I^{2} \leq 25 \%\right)$, moderate $\left(I^{2}>25 \%\right.$ and $\left.<75 \%\right)$, or high $\left(I^{2} \geq 75 \%\right)$ [24]. Additionally, Q-statistic was used to assess the presence of heterogeneity. $\mathrm{P}_{\mathrm{Q} \text { statistic }} \geq 0.05$ was considered to indicate no significant heterogeneity among the included studies. Study characteristics including geographic location, "primary" aim of the study, median number of participants, type of phytoestrogens, median duration of intervention, route of phytoestrogen administration, time since menopause onset, and women's health status (healthy vs. women with preexisting chronic disease/nonhealthy) were pre-specified as characteristics for assessment of heterogeneity and were evaluated using stratified analyses and randomeffects meta-regression, if 10 or more studies were included in the metaanalysis [25]. To assess the influence of each individual study to the overall estimates of the rest of the studies, leave-one-out sensitivity analysis was performed iteratively by removing one study at a time to confirm that the findings were not influenced by any single study. Publication bias was evaluated through a funnel plot and asymmetry was assessed using the Egger's test. All tests were two-tailed and pvalues of 0.05 or less was considered as statistically significant. STATA release 14 (Stata Corp, College Station, Texas) was used for all 


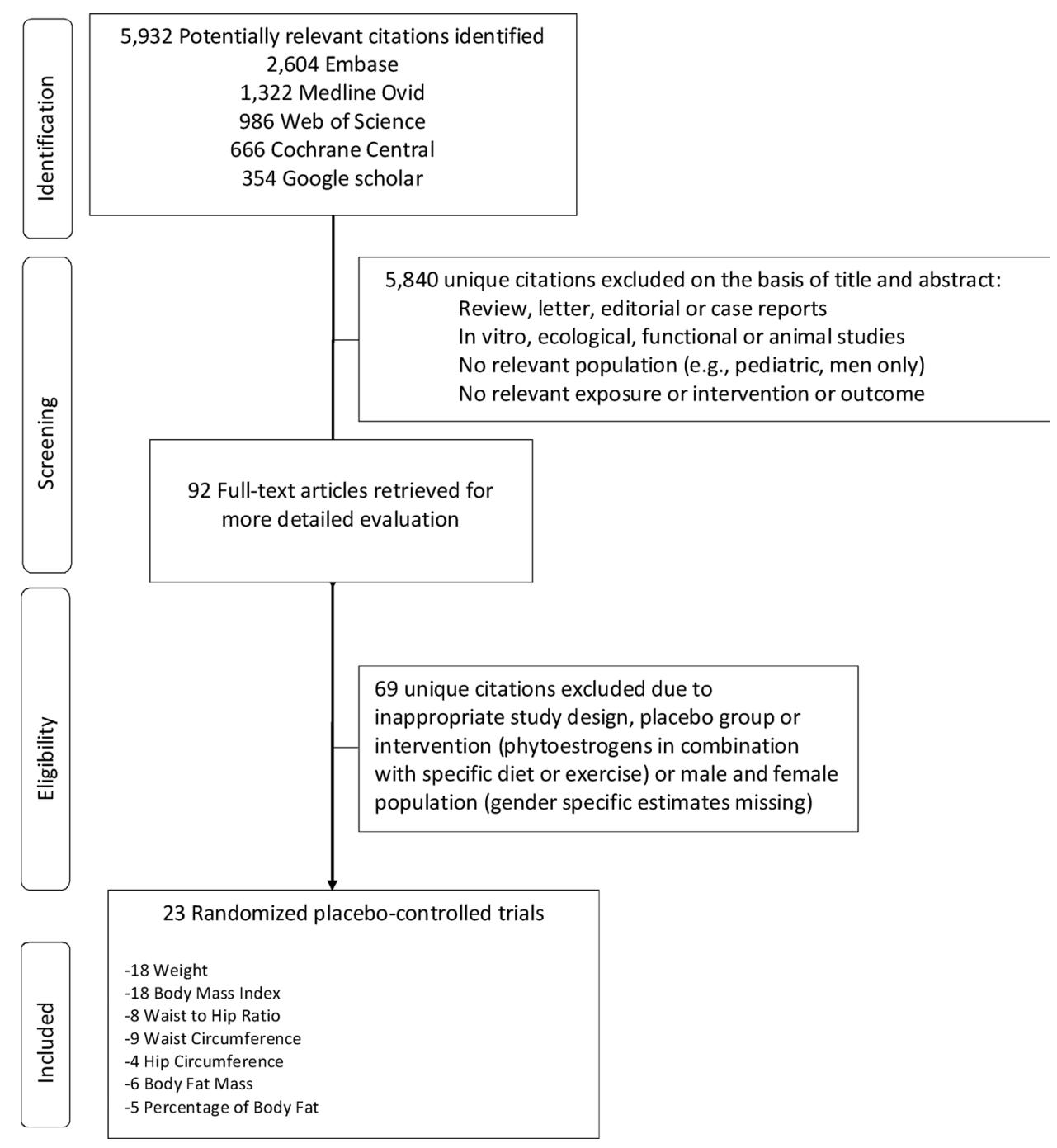

Fig. 1. Flowchart of studies included in this review.

statistical analyses.

\section{Results}

\subsection{Characteristics of included RCTs}

Overall 5932 references were identified using the search strategy. After initial screening based on titles and abstracts, the full texts of 92 articles were retrieved and evaluated further. As shown in Fig. 1, after full text assessment, 69 studies were excluded due to inappropriate study design, inappropriate population/exposure studies, or gender specific estimates missing. The remaining 23 RCTs [15-20,26-43] were included in the review and meta-analysis. In total 1880 postmenopausal women (1130 in intervention arm and 750 in placebo arm) were included in the meta-analysis of 23 RCTs. Six trials were done in Asia, 6 in Europe, 5 in North America and 6 in South America. Most of the studies were done among healthy women $(n=19)$, while four RCTs were conducted in women with metabolic syndrome, type 2 diabetes (T2D), sarcopenic obese, and one RCT in women with prehypertension. All women included in current review were postmenopausal and on average 4.19-15.9 years into menopause, and did not use MHT. The follow-up ranged from 8 to 48 weeks, with majority of RCTs lasting for 24 weeks $(n=11)$. In nine included RCTs, the effect of phytoestrogen supplementation on parameters of body composition was primarily investigated, while the rest of RCTs investigated the other outcomes (e.g. effect of phytoestrogen supplementation on bone mineral density, menopausal symptoms relief), but reported baseline and end study data on anthropometric parameters of interest. Detailed characteristics of included RCTs are presented in Table 1.

\subsection{Association between phytoestrogen supplementation and parameters of body composition}

Data from 18 RCTs, including 1692 postmenopausal women, contributed to the meta-analysis on effects of phytoestrogen supplementation on body weight. Consumption of any type of phytoestrogen supplements, as compared to placebo, was not associated with significant decrease in body weight in postmenopausal women [pooled mean difference of changes: $-0.14 \mathrm{~kg}$ (95\%CI: -0.49 to 0.21 ) (Fig. 2), in subgroup analysis, a significant decrease in body weight with overall phytoestrogens supplement use as compared to placebo was found in healthy postmenopausal women [pooled mean difference of changes: $-0.28 \mathrm{~kg}(95 \% \mathrm{CI}$ : -0.52 to -0.04$)$ ]. In contrast, phytoestrogen supplementation was associated with increased body weight in postmenopausal women with preexisting health disorders (prediabetes, T2D, prehypertension and hyperlipidemia), [pooled mean difference of changes: $0.78 \mathrm{~kg}$ (95\%CI: 0.53-1.03)] (Fig. 2).

Based on the findings of 18 RCTs, including 1456 postmenopausal women, overall phytoestrogen supplementation was not associated with BMI changes, [the pooled mean difference of BMI was $0.01 \mathrm{~kg} / \mathrm{m}^{2}$ 


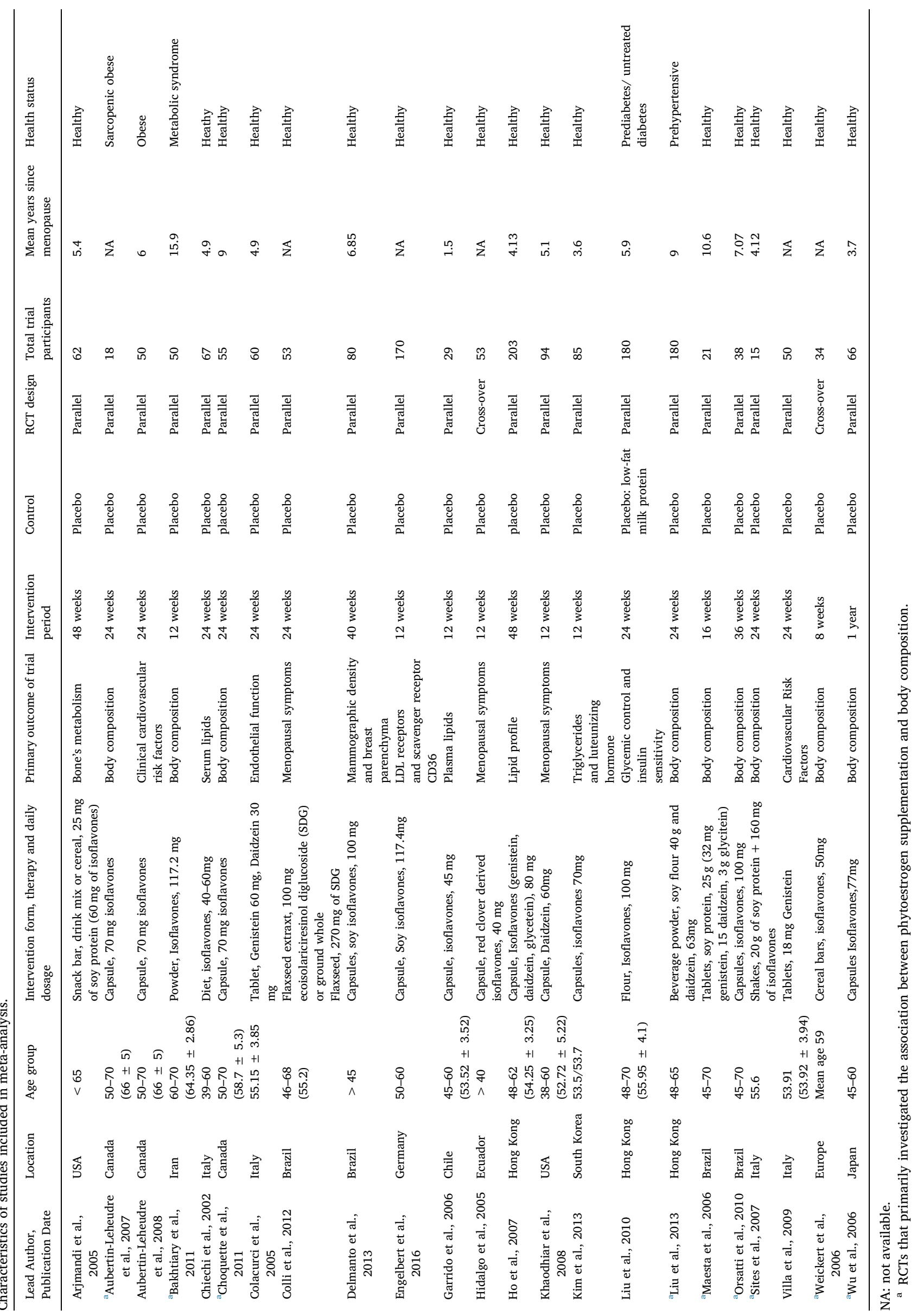




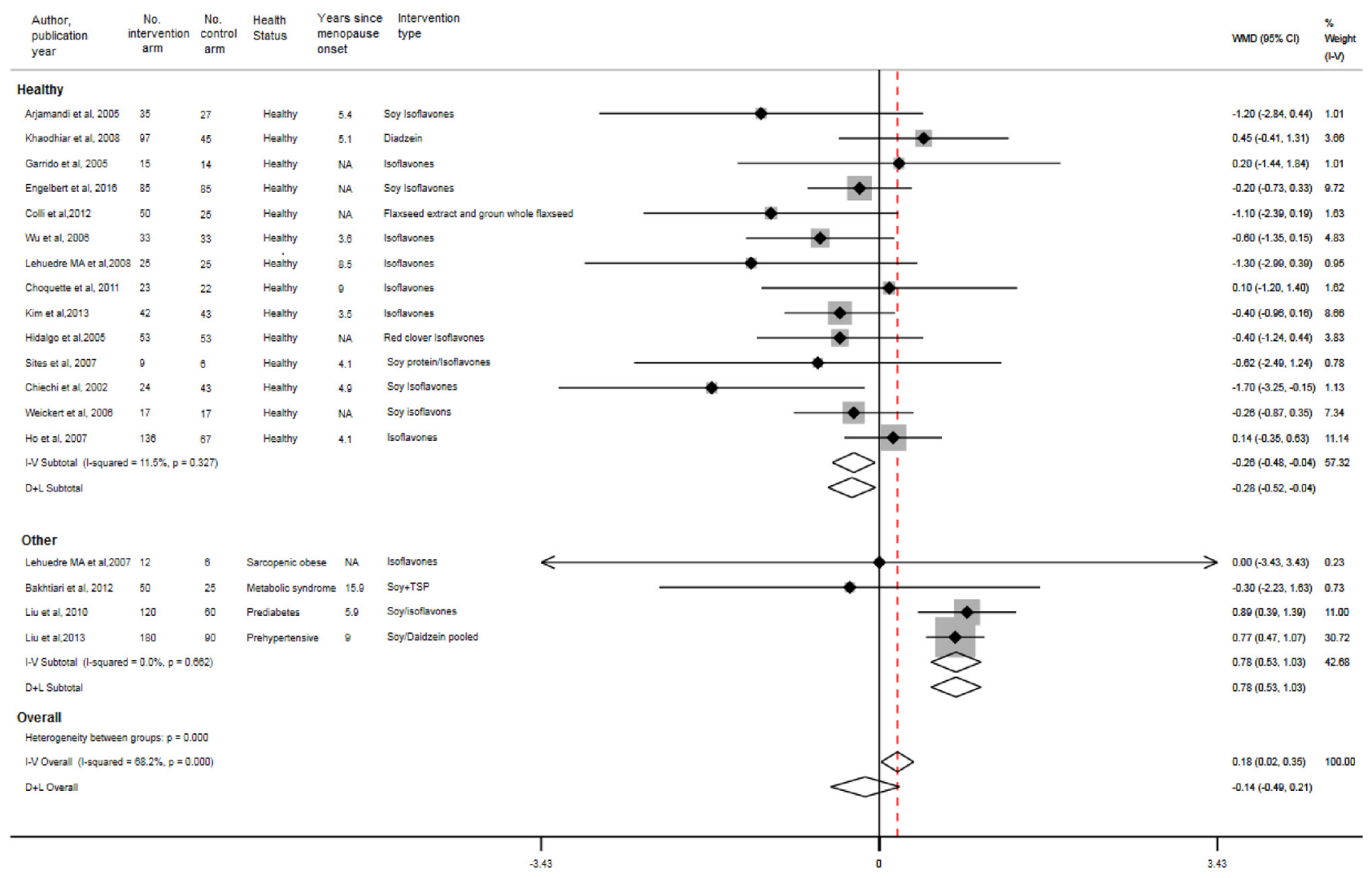

Fig. 2. The association between phytoestrogen supplementation and changes in body weight.

Pooled mean difference is based on 18 RCTs, including 1692 postmenopausal women (1006 in intervention arm and 686 in control arm).

WMD, weighted mean different. Mean difference refers to mean difference of changes between treatment groups. Size of data markers are proportional to the inverse of the variance of the effect estimate. P value comes from $Q$ statistics.

(95\%CI: -0.14 to 0.16 )] (Fig. 3). In subgroup analysis on health status, the results remained similar, with no significant decrease in mean difference of BMI among healthy and postmenopausal women with preexisting health conditions (Fig. 3). When stratified by type of phytoestrogen supplements, a significant increase was observed in body weight and BMI change with daidzein [pooled mean difference of changes: $0.92 \mathrm{~kg}$ (95\%CI: 0.24-1.59)] and [pooled mean difference of changes: $0.35 \mathrm{~kg} / \mathrm{m}^{2}$ (95\%CI: 0.17-0.52] respectively. Moreover, a significant decrease of body weight was found with isoflavone mix supplements [pooled mean difference of changes: $-0.24 \mathrm{Kg}(95 \% \mathrm{CI}$ : -0.46 to -0.01 )] (Table 2).

The data from 8 RCTs including 847 postmenopausal women contributed to the meta-analysis on effects of phytoestrogen supplementation on WHR. Pooled mean WHR change was slightly and significantly decreased, [pooled mean difference of changes of was $-0.01 \mathrm{~cm}$ (95\%CI: -0.01 to -0.006 ) (Fig. 4).

Data from 9 RCTs, including 824 postmenopausal women and 4 RCTs including 610 postmenopausal women investigated the association between phytoestrogen supplementation and waist and hip circumference changes, respectively. Consumption of any type of phytoestrogen supplements, as compared to placebo, was not associated with a reduction in changes of waist circumference and hip circumference [pooled mean differences of change were: $0.27 \mathrm{~cm}(95 \% \mathrm{CI}$ : -0.38 to 0.92$)$ and $0.49 \mathrm{~cm}(95 \% \mathrm{CI}-0.20$ to 1.17$)$ ] (Supplemental Figure 1). Furthermore, pooled mean differences based on 6 RCTs, including 421 and 5 RCTs including 573 postmenopausal women showed no association between overall phytoestrogen use and changes in the body fat and percentage of body fat. Pooled mean difference of changes were $-0.23 \mathrm{~kg}$ ( $95 \% \mathrm{CI}$ : -0.74 to 0.28 ) and $-0.26 \%$ (95\% CI -0.75 to 0.18), respectively (Supplemental Figure 2).

\subsection{Assessments of bias, heterogeneity and sensitivity analysis}

Two RCTs showed high risk of bias in two domains; however, for most of the RCTs $(n=21)$, the risk of bias could not be classified in one or more domains (Supplemental Table 2). The four of seven metaanalyses of RCTs showed high between-study heterogeneity, with an $\mathrm{I}^{2}$ estimate exceeding $75 \%$ and $\mathrm{P}_{\mathrm{Q} \text { statistic }}<0.05$, yet, in subgroup analysis by the type of phytoestrogens status, the heterogeneity varied from 0 to $97.2 \%$. In the current systematic review, as shown in the stratified analyses, high heterogeneity might be attributed to the study quality, differences in the methodology of trials and study location. Although $\mathrm{I}^{2}$ values varied across subgroup analysis, using "meta-regression method", it was not possible to explain the observed heterogeneity made by any of parameters investigated (the "primary" goal of the study, health status, median years since menopause, route of phytoestrogen administration, type of phytoestrogens, duration of the intervention, number of study participants nor by study location or study quality) (Table 1). However, when stratified by median number of trial participants, in small RCTs with median number of participants $\leq 66$ phytoestrogen supplementation was associated with significant decrease in body weight [pooled mean difference of changes $-0.49 \mathrm{~kg}$ (95\%CI: -0.87 to -0.11 )] (Table 2).

In a leave-one out sensitivity analysis, the pooled estimates were not influenced by any single study included in the analyzes (Supplemental Figure $3 \mathrm{a}-\mathrm{c}$ ). For the pooled analyses involving eight or more studies, publication bias was assessed visually using Begg's funnel plots. The funnel plot for pooled analysis of body weight changes was nonsymmetrical with Egger's $p$ value of 0.005 indicating presence of publication bias. However, funnel plots for pooled analyses reporting changes in BMI and WHR were approximately symmetrical with nonsignificant Egger's test estimates for all of these analyses ( $p$ values were 


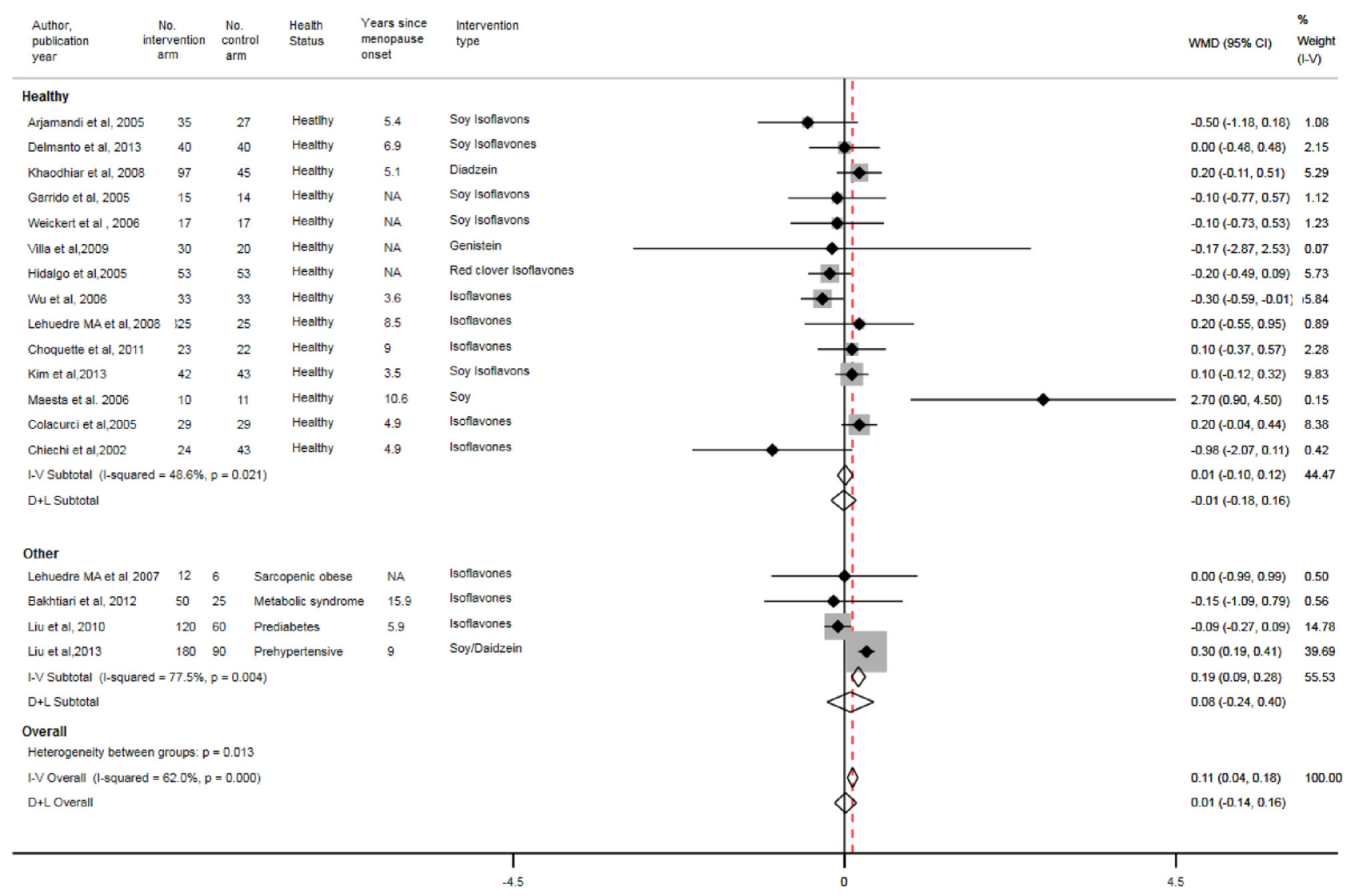

Fig. 3. The association between phytoestrogen intake and changes in BMI.

Pooled mean difference is based on 18 RCTs, including 1456 postmenopausal women (835 in intervention arm and 603 in control arm).

WMD, weighted mean different. Mean difference refers to mean difference of changes between treatment groups. Size of data markers are proportional to the inverse of the variance of the effect estimate. P value comes from $Q$ statistics.

0.19 and 0.97 respectively) (Fig. 5).

\section{Discussion}

\subsection{Summary of evidence}

In this systematic review and meta-analysis, phytoestrogen supplementation (compared to placebo and followed by usual diet) was associated with a slight decrease in WHR, and was not associated with changes in body weight, BMI, WC, HC, FM and PBF in postmenopausal women. There was also, an indication that type of phytoestrogens, and health status may play a role in modifying the effect of phytoestrogens in reducing body weight. For instance, a significant decrease in changes of body weight was observed among healthy postmenopausal women and when isoflavone mixture supplements were used. Contrary, body weight was increased in women with underlying metabolic disorders and/or with use of isoflavones rich in daidzein. Furthermore, trials with small sample size showed improved body weight effects in women associated with phytoestrogens, but this effect was not observed in trials with larger samples.

\subsection{Interpretation of findings}

In contrast to our findings, a meta-analysis published in 2013, reported a significant decrease in body weight with soy isoflavone supplementation [pooled mean difference of $-0.52 \mathrm{~kg}$ (95\%CI: -0.89 to -0.134)] [21]. Although, we did not observe overall significant associations between phytoestrogen supplementation and improvements in body composition parameters, we concluded that type of phytoestrogens and underlying metabolic status of women may play a role in modifying the effectiveness of phytoestrogens in reducing the body weight. There may be several factors that may have yield the differences in our findings on phytoestrogen supplementation and changes in body weight in comparison with previous review. First, we included all types of phytoestrogen supplements, while the prior meta-analysis investigated solely the effect of soy derived phytoestrogens and not the other types of phytoestrogen supplements (e.g. daidzein/genistein enriched formulations, soy products). Second, they included only nonAsian postmenopausal women, while in the current review seventeen non-Asian trials (European, North and South American) and six of Asian trials were included. Finally, the findings of the previous review were based on 9 RCTs and 578 postmenopausal women (272 in intervention trial and 256 in control arm) while we included 23 RCTs and 1880 postmenopausal women (1130 in intervention arm and 750 in placebo arm). Overall, there are some indications that certain types of phytoestrogens may be beneficial in reducing body weight, these findings are supported with experimental data and merit further investigation.

\subsection{Biological mechanisms/plausibility}

Adipose tissue is highly responsive to estrogen, and both, human and mouse fat tissue express estrogen receptor (ER) $\alpha$ and ER $\beta$ [44]. The mechanisms by which dietary soy and phytoestrogens may reduce adiposity are not fully understood. Phytoestrogens may affect the body composition directly binding estrogen receptors (mainly ER $\alpha$ ), by mediating the action of hormones thought to be involved in the regulation of body composition (adiponectin, ghrelin, insulin, leptin) or by altering the metabolic activity of adipocytes [45]. Indeed, experimental studies suggested that phytoestrogens could be useful in treating or preventing increased adiposity after menopause onset [46]. Findings 
Table 2

The subgroup analyses by study characteristics.

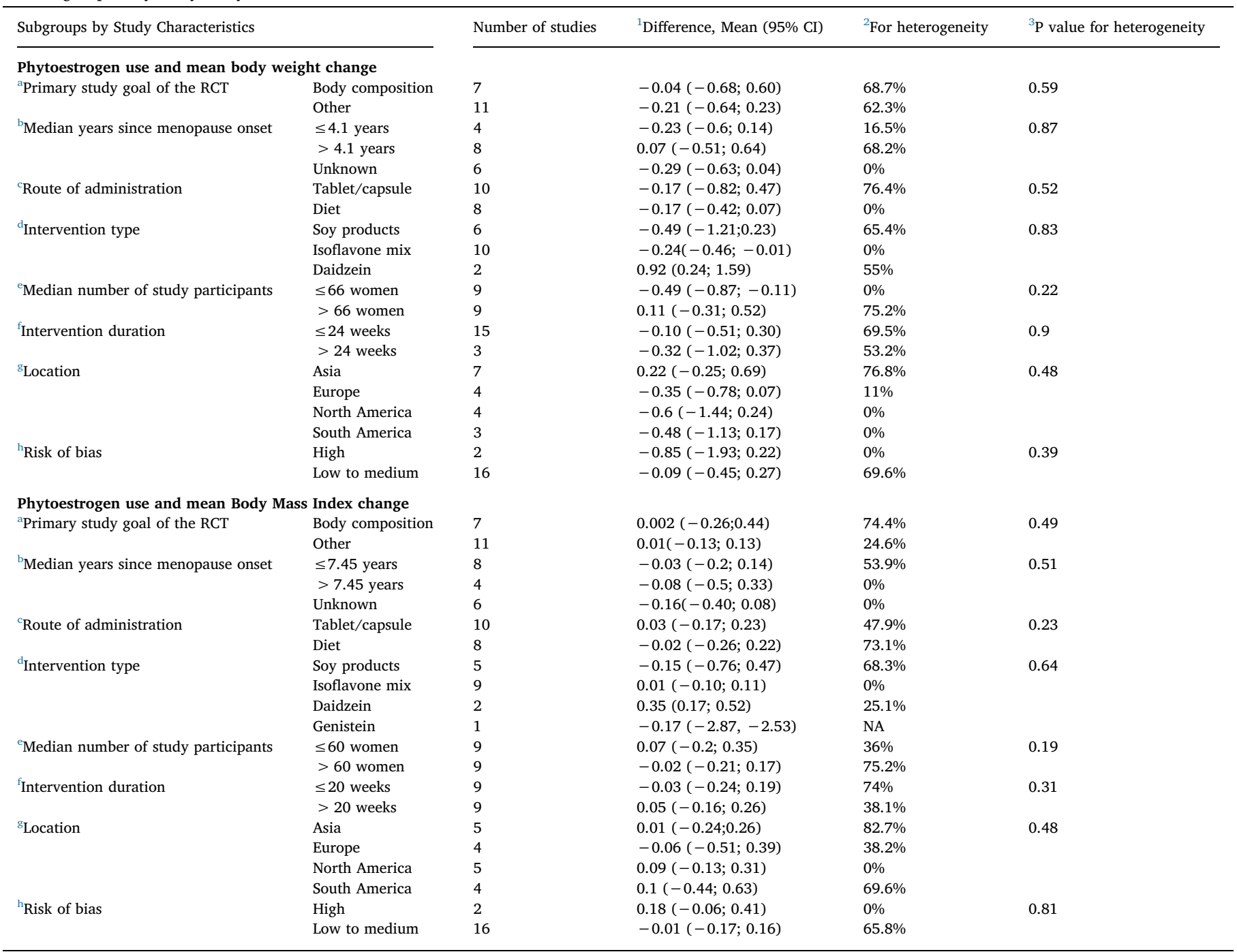

${ }^{1}$ Mean difference refers to mean difference of changes between treatment groups in body weight and BMI (subjects using phytoestrogens as compared with the subjects from control/placebo group).

${ }^{2}$ For heterogeneity was calculated using fixed- effects models.

${ }^{3} \mathrm{P}$ value for heterogeneity was evaluated using random-effects meta-regression (in case that more than 8 studies was meta-analyzed).

a Some of RCTs investigated the effect of phytoestrogens on body composition, the others investigated other outcomes but they reported changes in anthropometric parameters at baseline and at the end of the studies.

${ }^{\mathrm{b}}$ Median years since menopause, number of years since menopause onset, unknown: no information.

c Route of administration includes tablets/capsules use and other routes of administration (shake, powder, flower).

${ }^{d}$ Type of phytoestrogens includes use of soy derived isoflavones/soy protein + isoflavones, extracts of soy isoflavones/isoflavone mixture, daidzein/genistein supplements.

e Number of study participants: median number of participants calculated for each outcome separately.

${ }^{\mathrm{f}}$ Intervention duration: median intervention duration was calculated for each outcome separately.

${ }^{g}$ Location refers to study location, studies done in Asian ground and the other location (studies done in Europe, America and Australia).

${ }^{\mathrm{h}}$ Studies are judged to be at lower high risk of bias based on criteria to evaluate random sequence generation, allocation concealment, blinding of participants/ personnel and outcome assessment, incomplete outcome data and selective reporting.

from animal studies reported that fat abundance observed in mice exposed to dietary phytoestrogens correlated with enzymes which could be the key regulators of fatty acid oxidation (AMP-activated protein kinase and Acetyl-CoA carboxylase) [47]. Ovariectomized mice fed with a soy-rich diet have reduced weight and had less adipose deposition than those fed on a soy-free diet [48]. Genistein as the most abundant phytoestrogen in soy food, was extensively studied. Indeed, genistein reversed the truncal fat accumulation in ovariectomized rodent models $[44,46]$. Moreover, in vitro studies using isolated rat adipocytes genistein was found to inhibit the conversion of acetate into lipids, inhibit basal lipogenesis, inhibit the conversion of glucose to lipids more than estradiol, and increase basal lipolysis [49]. Although, genistein may cause adipose changes in mice in concentrations that are within the range of those reported in humans under various nutritional conditions, it is not clear whether genistein could have antilipogenic effects in humans. [44]. 


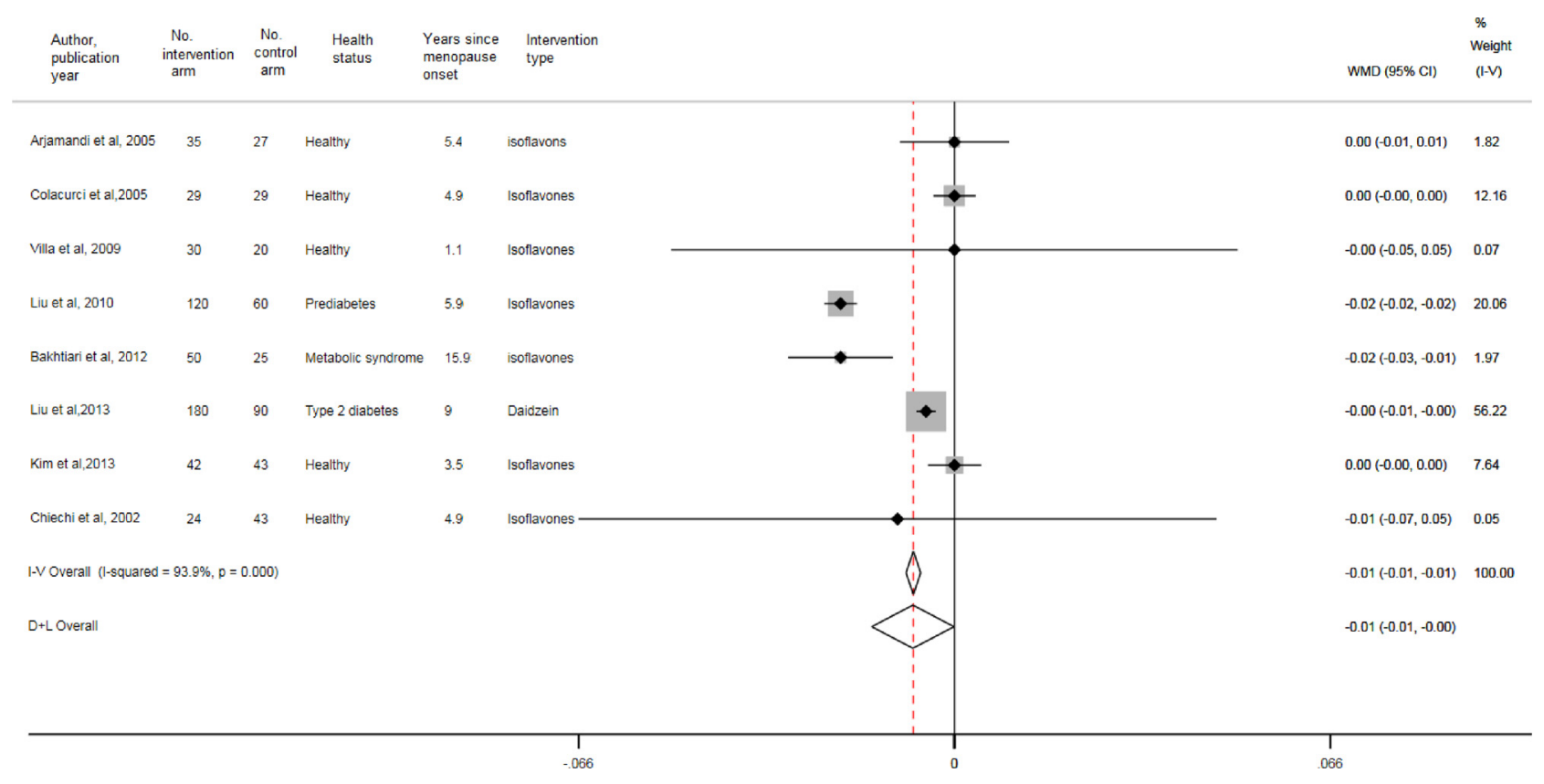

Fig. 4. The association between phytoestrogen supplementation and changes in waist to hip ratio.

Pooled mean difference is based on 8 RCTs, including 847 postmenopausal women (510 in intervention arm and 337 in control arm).

WMD, weighted mean different. Mean difference refers to mean difference of changes between treatment groups. Size of data markers are proportional to the inverse of the variance of the effect estimate. P value comes from Q statists.
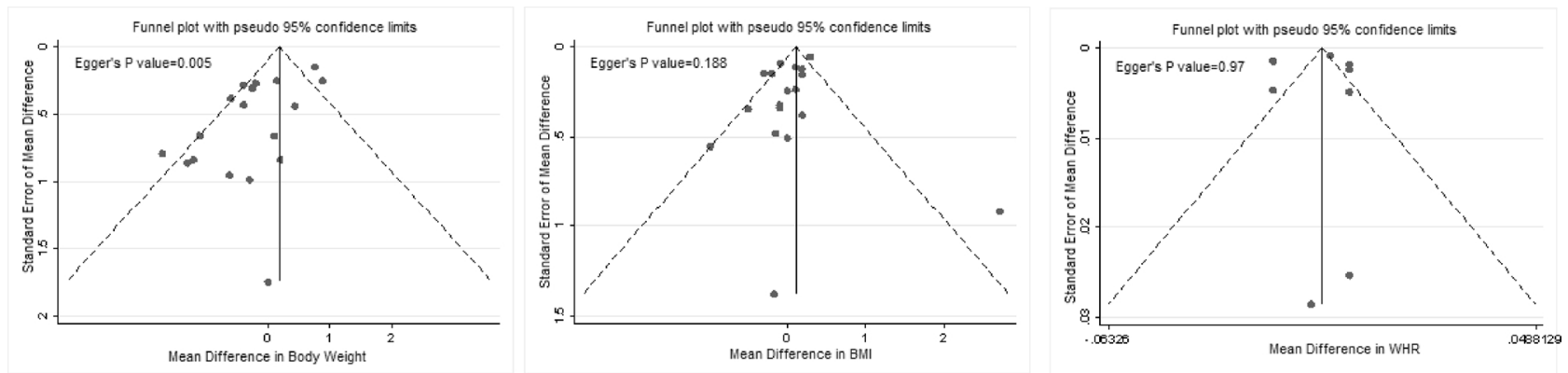

Fig. 5. Funnel plots for RCTs included in the main analysis.

The dotted lines show 95\% confidence intervals around the overall summary estimate calculated using a random effect model.

\subsection{Strengths and limitations}

To our knowledge, this is the first systematic review and metaanalysis including 1880 postmenopausal women to comprehensively address the associations of phytoestrogens with anthropometric parameters in women. Only clinical trials investigating phytoestrogen supplementation following usual/regular diet were included; therefore, studies that combined phytoestrogen supplementation with hypocaloric diets or in combination with exercise were not taken into consideration. Furthermore, we included clinical trials that did not "primary" investigate body composition but have reported outcomes of interest at baseline as well as at the end of trial. In subgroup analysis, those studies which did not primary investigate body composition were excluded and the results remained stable.

However, there are several limitations that need to be mentioned. First, in this study, we found a trend for the possibility of publication for studies investigating body weight changes. Despite the findings of funnel plots and Egger's test, the estimates could indicate minimal publication bias for RCTs investigating BMI and WHR, these approaches are potentially limited by their qualitative nature; therefore, findings on BMI and WHR may have been affected by publication bias as well. Second, the quality of included RCTs in this review was limited because the majority of included RCTs could not be classified in one or more domains, which might have contributed to the heterogeneity that has been observed in the meta-analyses presented in this study (quality and composition of supplements). Furthermore, in included trials women were on average 4.19-15.9 years into menopause. Early postmenopausal period is characterized by pronounced changes in body composition, with an increase in both overall and intra-abdominal adiposity [50]. In fact, in subgroup analysis by median time since menopause, pooled effect on body weight, although non-significant, was larger and in opposite direction compared to women with longer menopause duration. In addition, only four RCTs with more than 100 participants and only four RCT with a duration of the intervention of $\geq 6$ months were found, which might undermine the precision of the estimates and may limit the understanding of long-term effects of phytoestrogen supplementation on body composition in women. Considering these observations, the overall results of this study should be interpreted with caution. Finally, most of trials were published before 2013, with only one recent RCT investigating this topic after 2013. Thus, there may be some differences in formulation and quality of supplements of recent data compared to supplements was used $\geq 5$ years ago. 


\subsection{Scientific implications}

Inconsistent findings across different trials included in this review may be a consequence of variations in study protocols (differences in dose, duration, route of administration and composition of phytoestrogens used) and baseline characteristics of women studied (various comorbidities, years since menopause onset, the capacity of individuals to produce equol and the genetic susceptibility). Therefore, further well-designed clinical trials should clarify which type and dose of phytoestrogens may have favorable effect on body composition in women, in particular, soy protein isolates, isoflavone mixture and isolated genistein and daidzein effectiveness should be compared. The time since menopause onset, metabolic status and body composition at baseline should be the most important women's characteristics to account for when investigating the association between phytoestrogens and body composition. Additionally, due to variations in phytoestrogen metabolism among individuals, phytoestrogen metabolites (urinary concentrations) shall be measured to reduce measurement errors and address the issue of serum phytoestrogen concentrations over the study period.

\subsection{Conclusions}

The European Menopause and Andropause Society (EMAS) suggested non-hormonal management of menopausal symptoms as an option for women who cannot or do not wish to take MHT [9]. However, this review raises an important concern regarding the body weight changes with phytoestrogen supplementation. Based on current literature, phytoestrogen supplements followed by usual diet were not associated with changes in body weight. However, the type of phytoestrogens and underlying disease in women may play an important role in modifying the effectiveness of phytoestrogens in reducing body weight and may even lead to increase in body weight. Therefore, until obtaining more evidence in favor of beneficial role of phytoestrogens in reducing body weight, it might be much safer to combine phytoestrogen supplements (especially daidzein rich formulations) with hypocaloric diet/enhanced physical activity to maintain normal body weight during the supplementation period

\section{Contributors}

Marija Glisic contributed to analysis and interpretation, drafted and critically revised the manuscript.

Natyra Kastrati contributed to data acquisition, quality assessment and interpretation and critically revised the manuscript.

Juna Musa contributed to data acquisition, quality assessment and interpretation and critically revised the manuscript.

Jelena Milic contributed to interpretation and critically revised the manuscript.

Eralda Asllanaj contributed to interpretation and critically revised the manuscript.

Eliana Portilla Fernandez contributed to interpretation and critically revised the manuscript.

Jana Nano contributed to interpretation and critically revised the manuscript.

Carolina Ochoa Rosales contributed to interpretation and critically revised the manuscript.

Masoud Amiri contributed to interpretation and critically revised the manuscript.

Bledar Kraja contributed to interpretation and critically revised the manuscript.

Arjola Bano contributed to interpretation and critically revised the manuscript.

Wichor M. Bramer contributed to literature search and critically revised the manuscript.

Anton J.M. Roks participated in interpretation of the analyses and critically revised the manuscript.

A.H. Jan Danser participated in interpretation of the analyses and critically revised the manuscript.

Oscar H. Franco contributed to conception and design, contributed to analysis and interpretation and critically revised the manuscript.

Taulant Muka contributed to conception and design, contributed to analysis and interpretation and critically revised the manuscript. All authors gave the final approval and agree to be accountable for all aspects of work ensuring integrity and accuracy.

\section{Conflict of interest}

The authors declare that they have no conflict of interest.

\section{Funding}

No funding was received for the preparation of this review. Taulant Muka received research support from Metagenics. Inc., and currently works as pharmaceutical medicine physician at Novo Nordisk, Copenhagen, Denmark. Oscar H. Franco received grants/research support from Metagenics Inc. These funding sources had no role in the design and conduct of the study; collection, management, analysis, and interpretation of the data, or in the preparation, review or approval of the manuscript.

\section{Provenance and peer review}

This article has undergone peer review.

\section{References}

[1] S.R. Davis, I. Lambrinoudaki, M. Lumsden, G.D. Mishra, L. Pal, M. Rees, N. Santoro, T. Simoncini, Menopause, Nat. Rev. Dis. Primers 1 (2015) 15004.

[2] M.D. Dibonaventura, A. Chandran, M.A. Hsu, A. Bushmakin, Burden of vasomotor symptoms in France, Germany, Italy, Spain, and the United Kingdom, Int. J. Womens Health 5 (2013) 261-269.

[3] S.D. Reed, J.W. Lampe, C. Qu, G. Gundersen, S. Fuller, W.K. Copeland, K.M. Newton, Self-reported menopausal symptoms in a racially diverse population and soy food consumption, Maturitas 75 (2) (2013) 152-158.

[4] M.L. Misso, C. Jang, J. Adams, J. Tran, Y. Murata, R. Bell, W.C. Boon, E.R. Simpson, S.R. Davis, Differential expression of factors involved in fat metabolism with age and the menopause transition, Maturitas 51 (3) (2005) 299-306.

[5] R.C. Thurston, Y. Chang, P. Mancuso, K.A. Matthews, Adipokines, adiposity, and vasomotor symptoms during the menopause transition: findings from the study of women's health across the nation, Fertil. Steril. 100 (3) (2013) 793-800.

[6] C.J. Fernández-Alonso AM, P. Chedraui, M. Mendoza, A.M. Cuadros, F.R. PérezLópez, Obesity is related to increased menopausal symptoms among Spanish women, Menopause Int. 16 (3) (2010) 105-110.

[7] R.C. Thurston, M.R. Sowers, K. Sutton-Tyrrell, S.A. Everson-Rose, T.T. Lewis, D. Edmundowicz, K.A. Matthews, Abdominal adiposity and hot flashes among midlife women, Menopause 15 (2008) 429-434.

[8] R.C. Thurston, M.R. Sowers, B. Sternfeld, E.B. Gold, J. Bromberger, Y. Chang, H. Joffe, C.J. Crandall, L.E. Waetjen, K.A. Matthews, Gains in body fat and vasomotor symptom reporting over the menopausal transition: the study of women's health across the nation, Am. J. Epidemiol. 170 (6) (2009) 766-774.

[9] G. Mintziori, I. Lambrinoudaki, D.G. Goulis, I. Ceausu, H. Depypere, C.T. Erel, F.R. Perez-Lopez, K. Schenck-Gustafsson, T. Simoncini, F. Tremollieres, M. Rees, EMAS position statement: non-hormonal management of menopausal vasomotor symptoms, Maturitas 81 (3) (2015) 410-413.

[10] O.H. Franco, R. Chowdhury, J. Troup, T. Voortman, S. Kunutsor, M. Kavousi, C. Oliver-Williams, T. Muka, Use of plant-based therapies and menopausal symptoms: a systematic review and meta-analysis, JAMA 315 (23) (2016) 2554-2563.

[11] A. Cano, M.A. Garcia-Perez, J.J. Tarin, Isoflavones and cardiovascular disease, Maturitas 67 (3) (2010) 219-226.

[12] J.R. Guthrie, M. Ball, A. Murkies, L. Dennerstein, Dietary phytoestrogen intake in mid-life Australian-born women: relationship to health variables, Climacteric 3 (4) (2000) 254-261.

[13] Y. Yamori, Worldwide epidemic of obesity: hope for Japanese diets, Clin. Exp. 
Pharmacol. Physiol. 2 (Suppl. 31) (2004) S2-S4.

[14] D. Goodman-Gruen, D. Kritz-Silverstein, Usual dietary isoflavone intake and body composition in postmenopausal women, Menopause 10 (5) (2003) 427-432.

[15] S.C. Ho, Y.M. Chen, S.S. Ho, J.L. Woo, Soy isoflavone supplementation and fasting serum glucose and lipid profile among postmenopausal Chinese women: a doubleblind, randomized, placebo-controlled trial, Menopause 14 (5) (2007) 905-912.

[16] J. Kim, H. Lee, O. Lee, K.H. Lee, Y.B. Lee, K.D. Young, Y.H. Jeong, R. Choue, Isoflavone supplementation influenced levels of triglyceride and luteunizing hormone in Korean postmenopausal women, Arch. Pharmacol. Res. 36 (3) (2013) 306-313.

[17] C.K. Sites, B.C. Cooper, M.J. Toth, A. Gastaldelli, A. Arabshahi, S. Barnes, Effect of a daily supplement of soy protein on body composition and insulin secretion in postmenopausal women, Fertil. Steril. 88 (6) (2007) 1609-1617.

[18] B.H. Arjmandi, E.A. Lucas, D.A. Khalil, L. Devareddy, B.J. Smith, J. McDonald, A.B. Arquitt, M.E. Payton, C. Mason, One year soy protein supplementation has positive effects on bone formation markers but not bone density in postmenopausal women, Nutr. J. 4 (2005) 8.

[19] Z.M. Liu, S.C. Ho, Y.M. Chen, J. Woo, A six-month randomized controlled trial of whole soy and isoflavones daidzein on body composition in equol-producing postmenopausal women with prehypertension, J. Obes. 2013 (2013).

[20] N. Maesta, E.A.P. Nahas, J. Nahas-Neto, F.L. Orsatti, C.E. Fernandes, P. Traiman, R.C. Burini, Effects of soy protein and resistance exercise on body composition and blood lipids in postmenopausal women, Maturitas 56 (4) (2007) 350-358.

[21] Y.B. Zhang, W.H. Chen, J.J. Guo, Z.H. Fu, C. Yi, M. Zhang, X.L. Na, Soy isoflavone supplementation could reduce body weight and improve glucose metabolism in non-Asian postmenopausal women-a meta-analysis, Nutrition 29 (1) (2013) 8-14.

[22] D. Moher, A. Liberati, J. Tetzlaff, D.G. Altman, P. Group, Preferred reporting items for systematic reviews and meta-analyses: the PRISMA statement, PLoS Med. 6 (7) (2009) e1000097.

[23] J.P.T. Higgins, S. Green (Eds.), Cochrane Handbook for Systematic Reviews of Interventions Version 5.1.0 [updated March 2011], The Cochrane Collaboration, 2011Available from www.handbook.cochrane.org.

[24] J.P. Higgins, S.G. Thompson, J.J. Deeks, D.G. Altman, Measuring inconsistency in meta-analyses, BMJ 327 (7414) (2003) 557-560.

[25] S.G. Thompson, S.J. Sharp, Explaining heterogeneity in meta-analysis: a comparison of methods, Stat. Med. 18 (20) (1999) 2693-2708.

[26] M. Aubertin-Leheudre, C. Lord, A. Khalil, I.J. Dionne, Effect of 6 months of exercise and isoflavone supplementation on clinical cardiovascular risk factors in obese postmenopausal women: a randomized, double-blind study, Menopause 14 (4) (2007) 624-629.

[27] A. Bakhtiari, Z. Yassin, P. Hanachi, A. Rahmat, Z. Ahmad, P. Sajadi, S. Shojaei, Effects of soy on body composition: a 12-week randomized controlled trial among Iranian elderly women with metabolic syndrome, Iran. J. Public Health 41 (4) (2012) 9-18.

[28] S. Choquette, E. Riesco, E. Cormier, T. Dion, M. Aubertin-Leheudre, I.J. Dionne, Effects of soya isoflavones and exercise on body composition and clinical risk factors of cardiovascular diseases in overweight postmenopausal women: a 6-month double-blind controlled trial, Br. J. Nutr. 105 (8) (2011) 1199-1209.

[29] N. Colacurci, A. Chiàntera, F. Fornaro, V. De Novellis, D. Manzella, A. Arciello, V. Chiàntera, L. Improta, G. Paolisso, Effects of soy isoflavones on endothelial function in healthy postmenopausal women, Menopause 12 (3) (2005) 299-307.

[30] A. Garrido, M.P. De la Maza, S. Hirsch, L. Valladares, Soy isoflavones affect platelet thromboxane A2 receptor density but not plasma lipids in menopausal women, Maturitas 54 (3) (2006) 270-276.

[31] Z.M. Liu, Y.M. Chen, S.C. Ho, Y.P. Ho, J. Woo, Effects of soy protein and isoflavones on glycemic control and insulin sensitivity: a 6-mo double-blind, randomized, placebo-controlled trial in postmenopausal Chinese women with prediabetes or untreated early diabetes, Am. J. Clin. Nutr. 91 (5) (2010) 1394-1401.

[32] J. Wu, J. Oka, I. Tabata, M. Higuchi, T. Toda, N. Fuku, J. Ezaki, F. Sugiyama, S. Uchiyama, K. Yamada, Y. Ishimi, Effects of isoflavone and exercise on BMD and fat mass in postmenopausal Japanese women: a 1-year randomized placebo-controlled trial, J. Bone Miner. Res. 21 (5) (2006) 780-789.

[33] L.M. Chiechi, G. Secreto, A. Vimercati, P. Greco, E. Venturelli, F. Pansini, M. Fanelli,
P. Loizzi, L. Selvaggi, The effects of a soy rich diet on serum lipids: the Menfis randomized trial, Maturitas 41 (2) (2002) 97-104.

[34] P. Villa, B. Costantini, R. Suriano, C. Perri, F. Macrì, L. Ricciardi, S. Panunzi, A. Lanzone, The differential effect of the phytoestrogen genistein on cardiovascular risk factors in postmenopausal women: relationship with the metabolic status, J. Clin. Endocrinol. Metab. 94 (2) (2009) 552-558.

[35] M.R. Martin, O. Weickert, Bärbel Otto, Wendy L. Hall, Katherina Vafeiadou, Jesper Hallund, Marika Ferrari, Duncan Talbot, Francesco Branca, Susanne Bügel, Christine M. Williams, Hans-Joachim Zunft, Corinna Koebnick, Soy isoflavones increase preprandial peptide YY (PYY), but have no effect on ghrelin and body weight in healthy postmenopausal women, J. Negat. Results Biomed. (2006) 5-11.

[36] F.L. Orsatti, E.A. Nahas, J. Nahas-Neto, N. Maesta, C.L. Orsatti, C.E. Fernandes, Effects of resistance training and soy isoflavone on body composition in postmenopausal women, Obstet. Gynecol. Int. 2010 (2010) 156037.

[37] M. Aubertin-Leheudre, C. Lord, A. Khalil, I.J. Dionne, Isoflavones and clinical cardiovascular risk factors in obese postmenopausal women: a randomized doubleblind placebo-controlled trial, J. Womens Health (Larchmt.) 17 (8) (2008) 1363-1369.

[38] L. Khaodhiar, H.A. Ricciotti, L. Li, W. Pan, M. Schickel, J. Zhou, G.L. Blackburn, Daidzein-rich isoflavone aglycones are potentially effective in reducing hot flashes in menopausal women, Menopause 15 (1) (2008) 125-132.

[39] A. Delmanto, J. Nahas-Neto, P. Traiman, G. Uemura, E.C. Pessoa, E.A. Nahas, Effects of soy isoflavones on mammographic density and breast parenchyma in postmenopausal women: a randomized, double-blind, placebo-controlled clinical trial, Menopause 20 (10) (2013) 1049-1054.

[40] L.A. Hidalgo, P.A. Chedraui, N. Morocho, S. Ross, G. San Miguel, The effect of red clover isoflavones on menopausal symptoms, lipids and vaginal cytology in menopausal women: a randomized, double-blind, placebo-controlled study, Gynecol. Endocrinol. 21 (5) (2005) 257-264.

[41] A.K. Engelbert, S.T. Soukup, A. Roth, N. Hoffmann, D. Graf, B. Watzl, S.E. Kulling, A. Bub, Isoflavone supplementation in postmenopausal women does not affect leukocyte LDL receptor and scavenger receptor CD36 expression: a double-blind, randomized, placebo-controlled trial, Mol. Nutr. Food Res. 60 (9) (2016) 2008-2019.

[42] M.C. Colli, A. Bracht, A.A. Soares, A.L. de Oliveira, C.G. Boer, C.G. de Souza, R.M. Peralta, Evaluation of the efficacy of flaxseed meal and flaxseed extract in reducing menopausal symptoms, J. Med. Food 15 (9) (2012) 840-845.

[43] M.O. Weickert, M. Reimann, B. Otto, W.L. Hall, K. Vafeiadou, J. Hallund, M. Ferrari, D. Talbot, F. Branca, S. Bugel, C.M. Williams, H.J. Zunft, C. Koebnick, Soy isoflavones increase preprandial peptide YY (PYY), but have no effect on ghrelin and body weight in healthy postmenopausal women, J. Negat. Results Biomed. 5 (2006) 11.

[44] A. Naaz, S. Yellayi, M.A. Zakroczymski, D. Bunick, D.R. Doerge, D.B. Lubahn, W.G. Helferich, P.S. Cooke, The soy isoflavone genistein decreases adipose deposition in mice, Endocrinology 144 (8) (2003) 3315-3320.

[45] O.A. Matvienko, D.L. Alekel, U. Genschel, L. Ritland, M.D. Van Loan, K.J. Koehler, Appetitive hormones, but not isoflavone tablets, influence overall and central adiposity in healthy postmenopausal women, Menopause 17 (3) (2010) 594-601.

[46] H.K. Kim, C. Nelson-Dooley, M.A. Della-Fera, J.Y. Yang, W. Zhang, J. Duan, D.L. Hartzell, M.W. Hamrick, C.A. Baile, Genistein decreases food intake, body weight, and fat pad weight and causes adipose tissue apoptosis in ovariectomized female mice, J. Nutr. 136 (2) (2006) 409-414.

[47] C.R. Cederroth, S. Nef, Soy, phytoestrogens and metabolism: a review, Mol. Cell. Endocrinol. 304 (1-2) (2009) 30-42.

[48] J. Wu, X. Wang, H. Chiba, M. Higuchi, T. Nakatani, O. Ezaki, H. Cui, K. Yamada, Y. Ishimi, Combined intervention of soy isoflavone and moderate exercise prevents body fat elevation and bone loss in ovariectomized mice, Metabolism 53 (7) (2004) 942-948.

[49] K. Szkudelska, L. Nogowski, T. Szkudelski, Genistein affects lipogenesis and lipolysis in isolated rat adipocytes, J. Steroid Biochem. Mol. Biol. 75 (2000) 265-271.

[50] J.C. Lovejoy, C.M. Champagne, L. de Jonge, H. Xie, S.R. Smith, Increased visceral fat and decreased energy expenditure during the menopausal transition, Int. J. Obes. (Lond.) 32 (6) (2008) 949-958. 